\title{
Fuzzy-class point approach for software effort estimation using various adaptive regression methods
}

\author{
Shashank Mouli Satapathy • Mukesh Kumar • \\ Santanu Kumar Rath
}

Received: 2 May 2013/Accepted: 10 December 2013/Published online: 31 December 2013

(C) CSI Publications 2013

\begin{abstract}
The effort involved in developing a software product plays an important role in determining the success or failure. In the context of developing software using object oriented methodologies, traditional methods and metrics were extended to help managers in effort estimation activity. Software project managers require a reliable approach for effort estimation. It is especially important during the early stage of the software development life cycle. In this paper, the main goal is to estimate the cost of various software projects using class point approach and optimize the parameters using six types of adaptive regression techniques such as multi-layer perceptron, multivariate adaptive regression splines, projection pursuit regression, constrained topological mapping, $\mathrm{K}$ nearest neighbor regression and radial basis function network to achieve better accuracy. Also a comparative analysis of software effort estimation using these various adaptive regression techniques has been provided. By estimating the effort required to develop software projects accurately, we can have softwares with acceptable quality within budget and on planned schedules.
\end{abstract}

Keywords Software effort estimation - Class point . Fuzzy logic · MLP · MRS · PPR · CTM · KNN · RBFN $\cdot$ Object-oriented analysis and design

S. M. Satapathy $(\bowtie) \cdot$ M. Kumar $\cdot$ S. K. Rath

National Institute of Technology (NIT), Rourkela,

Rourkela, Orissa, India

e-mail: shashankamouli@gmail.com

\section{Introduction}

Software effort estimation is a major challenge in every software project. The project managers in the early phases of the development are very much indecisive about the future courses of action as the methodologies to be adopted are uncertain in nature. Object oriented technology is being presently accepted as the methodology for software development by researchers and practitioners. Several features offered by $\mathrm{OO}$ programming concept such as encapsulation, inheritance, polymorphism, abstraction, cohesion and coupling play an important role to manage systematically the development process [6]. Software size is one of the most important measures of a product. Traditional software estimation techniques like constructive cost estimation model (COCOMO) and function point analysis (FPA) have proven to be unsatisfactory for measuring the cost and effort of all types of software development, because the line of code and function point (FP) were both used for procedural programming concept [22]. The procedural oriented design splits the data and procedure, whereas the object oriented design combines both of them. Again, the effort estimation using FP approach can only be possible during coding phase of the software development life cycle. Hence there is a need to use some other technique to estimate the effort during early stage of the software development.

It is important to realize that the problem of learning/ estimation of dependencies from samples is only one part of the general experimental procedure used by researchers and practitioners who apply statistical methods such as fuzzy, machine learning etc. to draw conclusions from the data [9]. Hence to obtain good results in estimating software size, it is essential to consider the data obtained from previous projects. Need of accurate estimation has been 
always important while bidding for a contract or determining the feasibility of a project in terms of cost-benefit analysis. Since class is the basic logical unit of an object oriented system, the use of class point approach (CPA) to estimate the project effort helps to get a more accurate result. The CPA is calculated from the class diagram. Hence CPA can also be used to calculate the effort of the software project during design phase of the SDLC. There are two measures under CPA i.e. CP1 and CP2 used for class point size estimation process. The $\mathrm{CP} 1$ is calculated using two metrics i.e. number of external methods (NEM) and number of services requested (NSR), where as CP2 is calculated by using another metric in addition to NEM and NSR, i.e. number of attributes (NOA). The NEM measures the size of the interface of a class and is determined by the number of locally defined public methods. The NSR provides a measure of the interconnection of the system components. The NOA provides a measure of the NOA used in a class. In case of the FPA as well as CPA, the calculation of the technical complexity factor (TCF), depends on the influence factors of general system characteristics. But in CPA, non-technical factors such as management efficiency, developer's professional competence, security, reliability, maintainability and portability are not taken into consideration [28]. Hence in this paper, CPA has been extended to calculate the effort required to develop the software product while taking into consideration of these six non-technical factors. While calculating the number of class points, fuzzy logic has been applied to calculate the complexity of each class. Also in order to achieve better prediction accuracy, six different types of adaptive methods of regression techniques are used such as multi-layer perceptron (ANN), multivariate adaptive regression splines (MRS), projection pursuit regression (PPR), constrained topological mapping (CTM), K nearest neighbor regression $(\mathrm{KNN})$ and radial basis function network (RBFN). Finally a comparison of results obtained using these techniques have been provided to justify the result.

\section{Related work}

From the experiment over forty project data set, Costagliola et al. [10] have found that the prediction accuracy of CP1 is $75 \%$ and CP2 is $83 \%$. Zhou and Liu [28] have extended this approach by adding another measure named as CP3 based on CPA and have taken twenty-four system characteristics instead of eighteen considered by Gennaro Costagliola et al. By using this approach they found that the prediction accuracy of $\mathrm{CP} 1$ and $\mathrm{CP} 2$ remains unchanged. Kanmani et al. [19] have used the same CPA by using neural network in mapping the $\mathrm{CP} 1$ and $\mathrm{CP} 2$ into the effort and found that the prediction accuracy for $\mathrm{CP} 2$ is improved to $87 \%$.

Kanmani et al. [18] proposed another technique based on with fuzzy logic using subtractive clustering technique for calculating the effort and have compared the result with that obtained using the concept of artificial neural network (ANN). They found that fuzzy system using subtractive clustering technique yields better result than that of ANN. Kim et al. [20] introduced some new definitions of class point to increase understanding of a system's architectural complexity. They have taken the help of a number of extra parameters apart from NEM, NSR and NOA to calculate the total no of class points. Attarzadeh and Ow [2] described an enhanced soft computing model for the estimation of software cost and time estimation and compare the result with algorithmic model. Cherkassky et. al [7] use six representative methods implemented on artificial data sets to provide some insights on applicability of various methods. They conclude that no single method proved to be the best, since a method's performance depends significantly on the type of the target function (being estimated), and on the properties of training data (i.e., the number of samples, amount of noise, etc.).

Bors [4] introduced a few RBF training algorithms and showed how RBF networks can be applied for real-life applications. Sarimveis et al. [26] proposed a new algorithm for training RBF neural networks based on the subtractive clustering technique. Idri et al. [17] provide a comparison between a RBF neural network using C-means and a RBF neural network using APC-III, in terms of estimate accuracy, for software effort estimation based on COCOMO'81 dataset. Panchapakesan et al. [24] have used another approach to test how much one can reduce the error by changing the centers in an RBF network.

\section{Methodology used}

The following methodologies are used in this paper to calculate the effort of a software product.

\subsection{Class point analysis}

The CPA was introduced by Costagliola et al. in 1998 [11]. This was based on the FPA approach to represent the internal attributes of a software system in terms of counting. The idea underlying the CPA is the quantification of classes in a program in analogy to the function counting performed by the FP measure. This idea of Class Point Approach has been derived from the observation that in the procedural paradigm the basic programming units are functions or procedures; whereas, in the object-oriented paradigm, the logical building blocks are classes, which 
correspond to real-world objects and are related to each other. The Class Point size estimation process is structured into three main steps, corresponding to the FP approach i.e.

- Information processing size estimation

- Identification and classification of classes

- Evaluation of complexity level of each class

- Estimation of the total unadjusted class point

- Technical complexity factor estimation

- Final class point evaluation

During the first step, the design specifications are analysed in order to identify and classify the classes into four types of system components, namely the problem domain type (PDT), the human interaction type (HIT), the data management type (DMT), and the task management type (TMT) [10].

During the second step, each identified class is assigned a complexity level, which is determined on the basis of the local methods in the class and of the interaction of the class with the rest of the system. In some cases, the complexity level of each class is determined on the basis of the NEM, and the NSR. In some other cases, besides the above measures, the NOA measure is taken into account in order to evaluate the complexity level of each class. The block diagram shown in Fig. 1 explains the steps to calculate the class point.

For the calculation of CP1, the complexity level of the class is determined based on the value of NEM and NSR according to Table 1 . For example, if a class is having NEM value 7 and NSR value 3, then the complexity level assigned to the class is Average.

For the calculation of $\mathrm{CP} 2$, the complexity level of the class is determined based on the value of NEM, NOA and NSR according to Table $2 \mathrm{a}, \mathrm{b}$ and $\mathrm{c}$. In all these tables, NEM and NOA range varies with respect to the fixed NSR range.

Once a complexity level of each class has been assigned, such information and its type are used to assign a weight to the class given in Table 3. Then, the total unadjusted class point value (TUCP) is computed as a weighted sum.

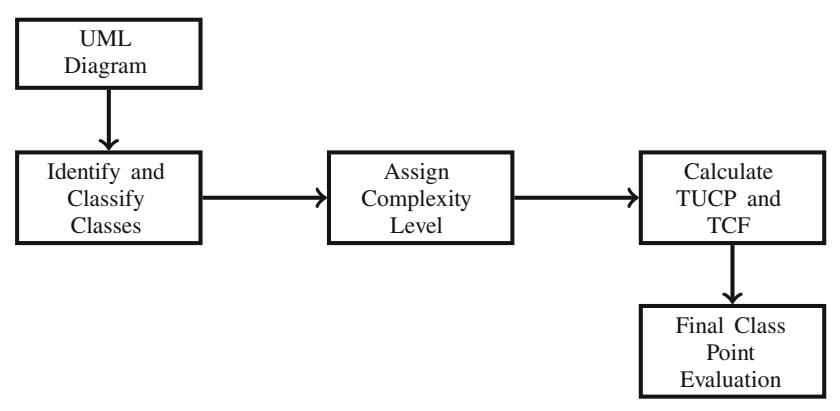

Fig. 1 Steps to calculate class point
Table 1 Complexity level evaluation for CP1

\begin{tabular}{lllll}
\hline & $0-4$ NEM & 5-8 NEM & 9-12 NEM & $\geq 13$ NEM \\
\hline $0-1$ NSR & Low & Low & Average & High \\
2-3 NSR & Low & Average & High & High \\
4-5 NSR & Average & High & High & Very high \\
$>5$ NSR & High & High & Very high & Very high \\
\hline
\end{tabular}

Table 2 Complexity level evaluation for CP2

\begin{tabular}{lllll}
\hline 0-2 NSR & 0-5 NOA & 6-9 NOA & 10-14 NOA & $\geq 15$ NOA \\
\hline (a) & & & & \\
0-4 NEM & Low & Low & Average & High \\
5-8 NEM & Low & Average & High & High \\
9-12 NEM & Average & High & High & Very high \\
$\geq 13$ NEM & High & High & Very high & Very high \\
\hline 3-4 NSR & 0-4 NOA & 5-8 NOA & 9-13 NOA & $\geq 14$ NOA \\
\hline
\end{tabular}

(b)

\begin{tabular}{lllll} 
0-3 NEM & Low & Low & Average & High \\
4-7 NEM & Low & Average & High & High \\
8-11 NEM & Average & High & High & Very high \\
$\geq 12$ NEM & High & High & Very high & Very high \\
\hline$\geq 5$ NSR & 0-3 NOA & $4-7$ NOA & 8-12 NOA & $\geq 13$ NOA \\
\hline c) & & & & \\
$0-2$ NEM & Low & Low & Average & High \\
3-6 NEM & Low & Average & High & High \\
7-10 NEM & Average & High & High & Very high \\
$\geq 11$ NEM & High & High & Very high & Very high \\
\hline
\end{tabular}

$\mathrm{TUCP}=\sum_{i=1}^{4} \sum_{j=1}^{4} w_{i j} \times x_{i j}$

where $x_{i j}$ is the number of classes of component type $i$ (problem domain, human interaction, etc.) with complexity level $j$ (low, average, high or very high), and $w_{i j}$ is the weighting value of type $i$ and complexity level $j$.

The TCF is determined by adjusting the TUCP with a value obtained by twenty-four different target software system characteristics, each on a scale of $0-5$. The sum of the influence degrees related to such general system characteristics forms the total degree of influence (TDI) as shown in Table 4, which is used to determine the TCF according to the following formula:

$\mathrm{TCF}=0.55+(0.01 * \mathrm{TDI})$

Finally, the adjusted class point (CP) value is determined by multiplying the total unadjusted class point (TUCP) value by TCF.

$C P=\mathrm{TUCP} * \mathrm{TCF}$ 
Table 3 Evaluation of TUCP for each class type

\begin{tabular}{llllll}
\hline System & Description & \multicolumn{2}{l}{ Complexity } \\
\cline { 3 - 6 } $\begin{array}{l}\text { component } \\
\text { type }\end{array}$ & Low & Average & High & $\begin{array}{c}\text { Very } \\
\text { high }\end{array}$ \\
\hline PDT & $\begin{array}{c}\text { Problem } \\
\text { domain type }\end{array}$ & 3 & 6 & 10 & 15 \\
HIT & $\begin{array}{c}\text { Human } \\
\text { interaction type }\end{array}$ & 4 & 7 & 12 & 19 \\
DMT & $\begin{array}{c}\text { Data management } \\
\text { type }\end{array}$ & 5 & 8 & 13 & 20 \\
TMT & $\begin{array}{c}\text { Task management } \\
\text { type }\end{array}$ & 4 & 6 & 9 & 13 \\
\hline
\end{tabular}

Table 4 Degree of Influences of 24 general system characteristics

\begin{tabular}{|c|c|c|c|c|c|}
\hline ID & $\begin{array}{l}\text { System } \\
\text { characteristics }\end{array}$ & DI & ID & System characteristics & DI \\
\hline $\mathrm{C} 1$ & $\begin{array}{l}\text { Data } \\
\text { communication }\end{array}$ & - & $\mathrm{C} 13$ & Multiple sites & - \\
\hline $\mathrm{C} 2$ & $\begin{array}{r}\text { Distributed } \\
\text { functions }\end{array}$ & - & $\mathrm{C} 14$ & Facilitation of change & - \\
\hline $\mathrm{C} 3$ & Performance & - & $\mathrm{C} 15$ & User adaptivity & - \\
\hline $\mathrm{C} 4$ & $\begin{array}{l}\text { Heavily used } \\
\text { configuration }\end{array}$ & - & $\mathrm{C} 16$ & Rapid prototyping & - \\
\hline $\mathrm{C} 5$ & Transaction rate & - & $\mathrm{C} 17$ & Multiuser interactivity & - \\
\hline C6 & Online data entry & - & $\mathrm{C} 18$ & Multiple interfaces & - \\
\hline $\mathrm{C} 7$ & $\begin{array}{l}\text { End-user } \\
\text { efficiency }\end{array}$ & - & $\mathrm{C} 19$ & Management efficiency & - \\
\hline $\mathrm{C} 8$ & Online update & - & $\mathrm{C} 20$ & $\begin{array}{l}\text { Developers' professional } \\
\text { competence }\end{array}$ & - \\
\hline $\mathrm{C} 9$ & $\begin{array}{l}\text { Complex } \\
\text { processing }\end{array}$ & - & $\mathrm{C} 21$ & Security & - \\
\hline $\mathrm{C} 10$ & Reusability & - & $\mathrm{C} 22$ & Reliability & - \\
\hline $\mathrm{C} 11$ & Installation ease & - & $\mathrm{C} 23$ & Maintainability & - \\
\hline $\mathrm{C} 12$ & Operational ease & - & $\mathrm{C} 24$ & Portability & - \\
\hline TDI & \multicolumn{4}{|c|}{ Total degree of influence (TDI) } & - \\
\hline
\end{tabular}

The final optimized class point value is then taken as input argument to those six adaptive regression models to estimate normalized effort.

\subsection{Multi-layer perceptron (MLP) technique}

MLP technique uses a feed forward ANN with one or more layers between input and output layer. Feed forward means that data flows in one direction from input to output layer (forward). This type of network is trained with the back propagation learning algorithm. MLPs are widely used for pattern classification, recognition, prediction and approximation. MLP technique can solve problems which are not linearly separable.
3.3 Multivariate adaptive regression splines (MRS) method

Multivariate Adaptive Regression Splines (MRS) method is presented by Friedman [14] in 1991 for flexible regression modeling of high dimensional data. The model takes the form of an expansion in product spline basis functions, where the number of basis function as well as the parameters associated with each one (product degree and knot locations) are automatically determined by the data. This procedure is motivated by the recursive partitioning approach to regression and shares its attractive properties. It is a non-parametric regression technique and can be seen as an extension of linear models that automatically model non-linearities and interactions between variables.

\subsection{Projection pursuit regression (PPR) method}

PPR is a method for nonparametric multiple regression developed by Friedman and Stuetzle [15] in the year 1981. It is an extension of an extension of additive models. This procedure models the regression surface as a sum of general smooth functions of linear combinations of the predictor variables in an iterative manner. It is more general than standard stepwise and stage-wise regression procedures, does not require the definition of a metric in the predictor space, and lends itself to graphical interpretation.

\subsection{Constrained topological mapping (CTM) method}

CTM method is proposed by Cherkassky and Lari-Najafi [8] in the year 1991. It is a modification of the original Kohonen's algorithm for self-organizing maps. CTM algorithm is proposed for regression analysis applications. Given a number of data points in N-dimensional input space, the proposed algorithm performs correct topological mapping of units (as the original algorithm) and at the same time preserves topological ordering of projections of these units onto (N-1)-dimensional subspace of independent coordinates.

\section{6 $\mathrm{K}$ nearest neighbor regression $(\mathrm{KNN})$ method}

KNN method is presented by Devroye [12] in the year 1978. In pattern recognition, the KNN is a method for classifying objects based on closest training examples in the feature space. It is amongst the simplest of all machine learning algorithms: an object is classified by a majority vote of its neighbors, with the object being assigned to the class most common amongst its $\mathrm{k}$ nearest neighbors $(\mathrm{k}$ is a positive integer, typically small). If $\mathrm{k}=1$, then the object is simply assigned to the class of its nearest neighbor. The same method can be used for regression, by simply 
assigning the property value for the object to be the average of the values of its $\mathrm{k}$ nearest neighbors.

\subsection{Radial basis function network (RBFN) method}

The method using radial basis function emerged as a variant of ANN in late 1980s [4]. The idea of RBFN derives from the theory of function approximation. The architecture of the RBFN is quite simple. An input layer consisting of sources node; a hidden layer in which each neuron computes its output using a radial basis function, that being in general a Gaussian function, and an output layer that builds a linear weighted sum of hidden neuron outputs and supplies the response of the network (effort). An RBFN has only output neuron. It can be modeled as:

$F(x)=\sum_{j=1}^{L} w_{j} \phi_{j}\left(\left\|x-c_{j}\right\|\right)$

where $\mathrm{L}$ is the number of hidden neurons, $x \in R^{p}$ is the input, $w_{j}$ are the output layer weights of the $\operatorname{RBFN}$ and $\phi(x)$ is Gaussian radial basis function given by:

$\phi_{j}\left(\left\|x-c_{j}\right\|\right)=\exp \left(-\frac{\left\|x-c_{j}\right\|^{2}}{\left(\sigma_{j}\right)^{2}}\right)$

where $\|$.$\| denotes the Euclidean distance, c_{j} \in R^{p}$ is the centre of the $j$ th hidden neuron and $\sigma_{j}^{2}$ is the width of the jth hidden neuron.

\section{Proposed approach}

The proposed work is based on data derived from forty student projects [10] developed using Java language and intends to evaluate software development effort. The use of such data in the validation process has provided initial experimental evidence of the effectiveness of the CPA. These data are used for the implementation of six adaptive methods for regression such as MLP, MRS, PPR, CTM, $\mathrm{KNN}$ and RBFN system model. The calculated result is then compared to measure the accuracy of the models. To calculate the effort of a given software project, basically the following steps have been used.

\section{Steps in effort estimation}

1. Data collection The data has been collected from previously developed projects.

2. Calculate class point The class point will be calculated as per the steps described in Fig. 1.

3. Select data The generated CP2 value in Step-2 has been used as input arguments.

4. Normalize dataset Input feature values were normalized over the range $[0,1]$. Let $\mathrm{X}$ be the dataset and $x$ is an element of the dataset, then the normalization of the $x$ can be calculated as :

$\operatorname{Normalized}(x)=\frac{x-\min (X)}{\max (X)-\min (X)}$

where

$\min (X)=$ the minimum value of the dataset $X$.

$\max (X)=$ the maximum value of the dataset $X$.

if $\max (X)$ is equal to $\min (X)$, then $\operatorname{Normalized}(x)$ is set to 0.5 .

5. Division of dataset Divide the number of data into three parts i.e. learning set, validation set and test set.

6. Perform model selection In this step, a fivefold cross validation is implemented for model selection. The model which provides the least NRMSE value than the other generated models based on the minimum validation and prediction error criteria has been selected to perform other operations.

7. Select best model By taking the average of all the fivefold's corresponding validation and prediction error (NRMSE) value, the best model has been selected. Finally the model has been plotted using training sample and testing sample.

Once the model is ready, the parameter of any new project can be given, and it will generate the estimated effort as output for that project.

\section{Experimental details}

In this paper to implement the proposed approach, dataset given in Table 5 has been used. In the data set, every row displays the details of one project developed in JAVA language, indicating values of NEM, NSR and NOA for that project. Apart from that, it also displays values of CP1, $\mathrm{CP} 2$ and the actual effort (denoted by EFH) expressed in terms of person-hours required to successfully complete the project.

\subsection{Calculate complexity of a class using fuzzy logic}

In the calculation of CP, Mamdani-type FIS has been used because Mamdani method is widely accepted for capturing expert knowledge. It allows us to describe the expertise in more intuitive and human-like manner. However, Mamdani-type FIS entails a substantial computational burden.

The model has three inputs i.e. NEM, NOA and NSR; and one output i.e. CP calculation as shown in Fig. 2. The main processes of this system include four activities: fuzzification, fuzzy rule base, fuzzy inference engine and defuzzification. All the input variables in this model are 
Table 5 Forty project dataset

\begin{tabular}{|c|c|c|c|c|c|c|}
\hline & $\mathrm{EFH}$ & $\mathrm{CP} 1$ & $\mathrm{CP} 2$ & NEM & NSR & NOA \\
\hline 1 & 286 & 103.18 & 110.55 & 142 & 97 & 170 \\
\hline 2 & 396 & 278.72 & 242.54 & 409 & 295 & 292 \\
\hline 3 & 471 & 473.90 & 446.60 & 821 & 567 & 929 \\
\hline 4 & 1016 & 851.44 & 760.96 & 975 & 723 & 755 \\
\hline 5 & 1261 & 1263.12 & 1242.60 & 997 & 764 & 1145 \\
\hline 6 & 261 & 196.68 & 180.84 & 225 & 181 & 400 \\
\hline 7 & 993 & 178.80 & 645.60 & 589 & 944 & 402 \\
\hline 8 & 552 & 213.30 & 208.56 & 262 & 167 & 260 \\
\hline 9 & 998 & 1095.00 & 905.00 & 697 & 929 & 385 \\
\hline 10 & 180 & 116.62 & 95.06 & 71 & 218 & 77 \\
\hline 11 & 482 & 267.80 & 251.55 & 368 & 504 & 559 \\
\hline 12 & 1083 & 687.57 & 766.29 & 789 & 362 & 682 \\
\hline 13 & 205 & 59.64 & 64.61 & 79 & 41 & 98 \\
\hline 14 & 851 & 697.48 & 620.10 & 542 & 392 & 508 \\
\hline 15 & 840 & 864.27 & 743.49 & 701 & 635 & 770 \\
\hline 16 & 1414 & 1386.32 & 1345.40 & 885 & 701 & 1087 \\
\hline 17 & 279 & 132.54 & 74.26 & 97 & 387 & 65 \\
\hline 18 & 621 & 550.55 & 481.66 & 382 & 654 & 293 \\
\hline 19 & 601 & 539.35 & 474.95 & 387 & 845 & 484 \\
\hline 20 & 680 & 489.06 & 438.90 & 347 & 870 & 304 \\
\hline 21 & 366 & 287.97 & 262.74 & 343 & 264 & 299 \\
\hline 22 & 947 & 663.60 & 627.60 & 944 & 421 & 637 \\
\hline 23 & 485 & 397.10 & 358.60 & 409 & 269 & 451 \\
\hline 24 & 812 & 678.28 & 590.42 & 531 & 401 & 520 \\
\hline 25 & 685 & 386.31 & 428.18 & 387 & 297 & 812 \\
\hline 26 & 638 & 268.45 & 280.84 & 373 & 278 & 788 \\
\hline 27 & 1803 & 2090.70 & 1719.25 & 724 & 1167 & 1633 \\
\hline 28 & 369 & 114.40 & 104.50 & 192 & 126 & 177 \\
\hline 29 & 439 & 162.87 & 156.64 & 169 & 128 & 181 \\
\hline 30 & 491 & 258.72 & 246.96 & 323 & 195 & 285 \\
\hline 31 & 484 & 289.68 & 241.40 & 363 & 398 & 444 \\
\hline 32 & 481 & 480.25 & 413.10 & 431 & 362 & 389 \\
\hline 33 & 861 & 778.75 & 738.70 & 692 & 653 & 858 \\
\hline 34 & 417 & 263.72 & 234.08 & 345 & 245 & 389 \\
\hline 35 & 268 & 217.36 & 195.36 & 218 & 187 & 448 \\
\hline 36 & 470 & 295.26 & 263.07 & 250 & 512 & 332 \\
\hline 37 & 436 & 117.48 & 126.38 & 135 & 121 & 193 \\
\hline 38 & 428 & 146.97 & 148.35 & 227 & 147 & 212 \\
\hline 39 & 436 & 169.74 & 200.10 & 213 & 183 & 318 \\
\hline 40 & 356 & 112.53 & 110.67 & 154 & 83 & 147 \\
\hline
\end{tabular}

changed to the fuzzy variables based on the fuzzification process. The complexity levels such as Low, Average, High, and Very High are defined for NOA and NEM variables, for different number of services requested (NSR). The steps to calculate the class point using FIS is shown below.
1. Different UML diagrams of a project may be fixed.

2. Each class has been classified into various domains such as PDT / HIT / DMT / TMT.

3. Then the no. of external methods (NEM), no. of services requested (NSR) and no. of attributes (NOA) from UML diagram has been extracted

4. Each classes has been assigned different complexity levels such as low, average, high and very high using K-means clustering algorithm basing on their NEM, NSR and NOA value as shown in Table 2.

5. The numeric value of complexity level assigned to a class found in Step-4 has been calculated using fuzzy logic.

6. Then the unadjusted class point (UCP) has been calculated by multiplying the numeric value got in Step-5 and its corresponding value shown in Table 3.

7. The total unadjusted class point (TUCP) has been calculated by adding the UCP of all classes given in Eq. 1 defined in Sect. 3.1

8. Then total complexity factor (TCF) has been calculated by using the Eq. 2 defined in Sect. 3.1

9. Finally the final $\mathrm{CP}$ count has been calculated by using the Eq. 3 defined in Sect. 3.1

\subsubsection{Results and discussion}

In this paper, a fuzzy set for each linguistic value with a triangular membership function (TRIMF) has been defined, which is shown in Fig. 3.

This figure shows the triangular membership function for the three inputs i.e. NEM, NOA and NSR and the output i.e. CP. The fuzzy sets corresponding to the various associated linguistic values for each type of inputs have been defined. The proposed fuzzy rules contain the linguistic variables related to the project. It is important to note that these rules were adjusted or calibrated, as well as all pertinent level functions, in accordance with the tests and the characteristics of the project. The number of rules those have been used in model are 48 for all input variables.

5.1.1.1 Fuzzy logic rules If NSR is low, NEM is low and NOA is low, then CP is LOW.

If NSR is low, NEM is low and NOA is average, then $\mathrm{CP}$ is LOW.

If NSR is high, NEM is very high and NOA is very high, then CP is VERYHIGH.

Rather than classifying the classes into four complexity levels i.e. Low, Average, High and Very High, the classes 


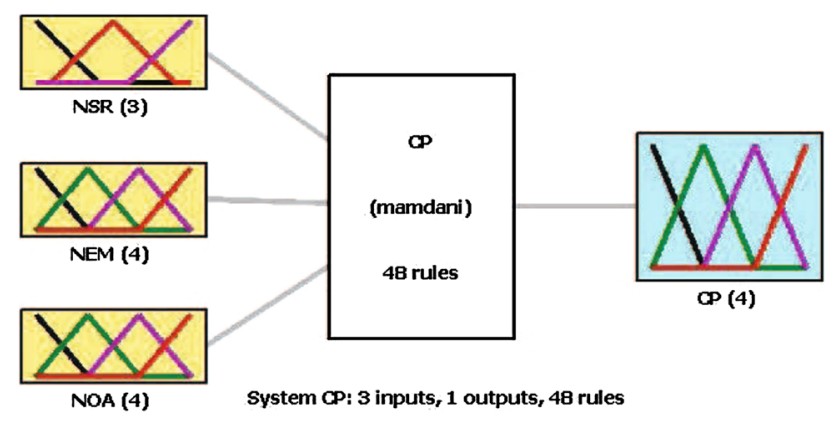

Fig. 2 Fuzzy inference system for class point calculation
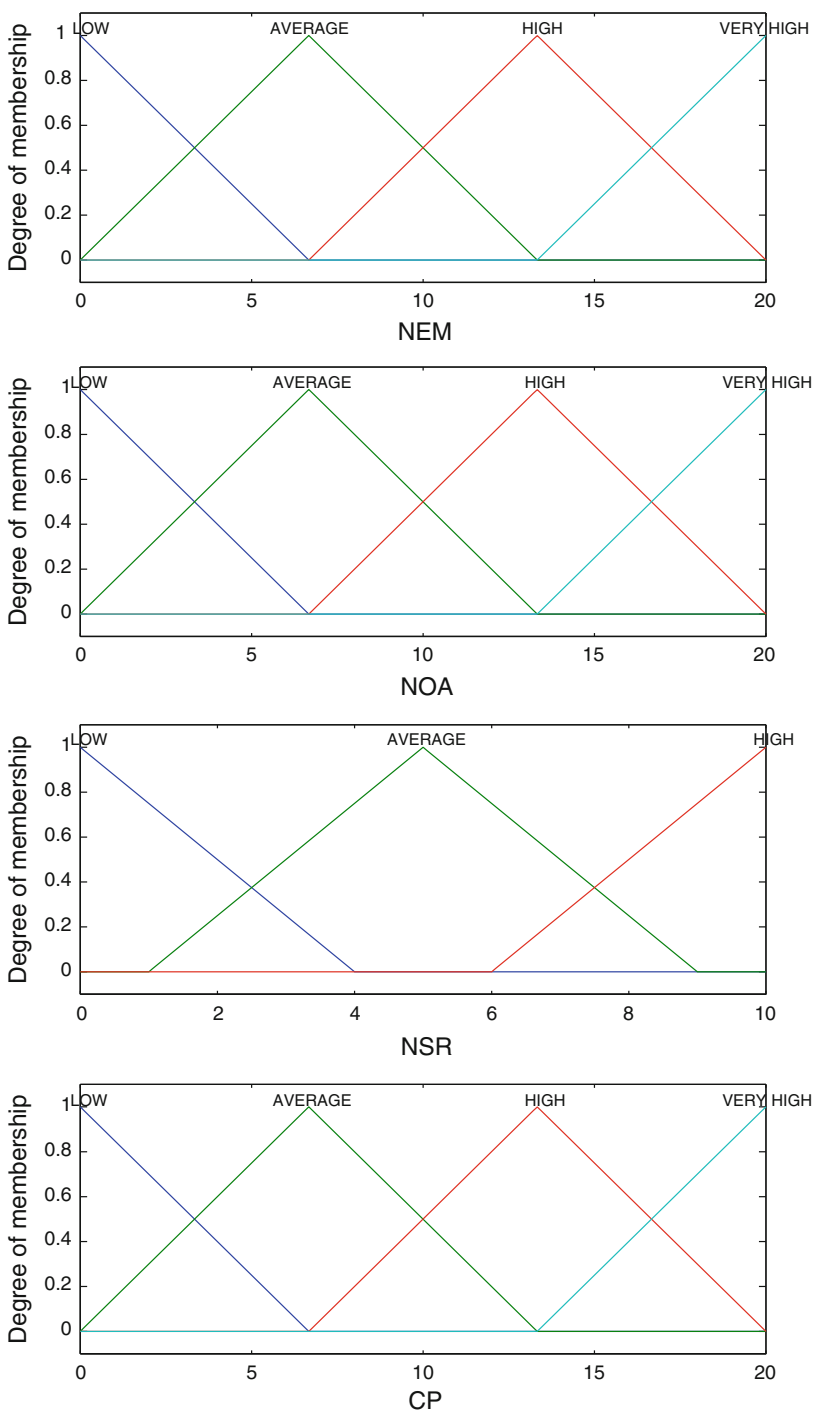

Fig. 3 Membership function for weight matrix of NEM, NOA, NSR and $\mathrm{CP}$

may be classified into 48 categories according to the number of transactions per classes. Hence 48 rules generated to calculate the weight associated with the class. The MATLAB fuzzy inference system (FIS) was used in the
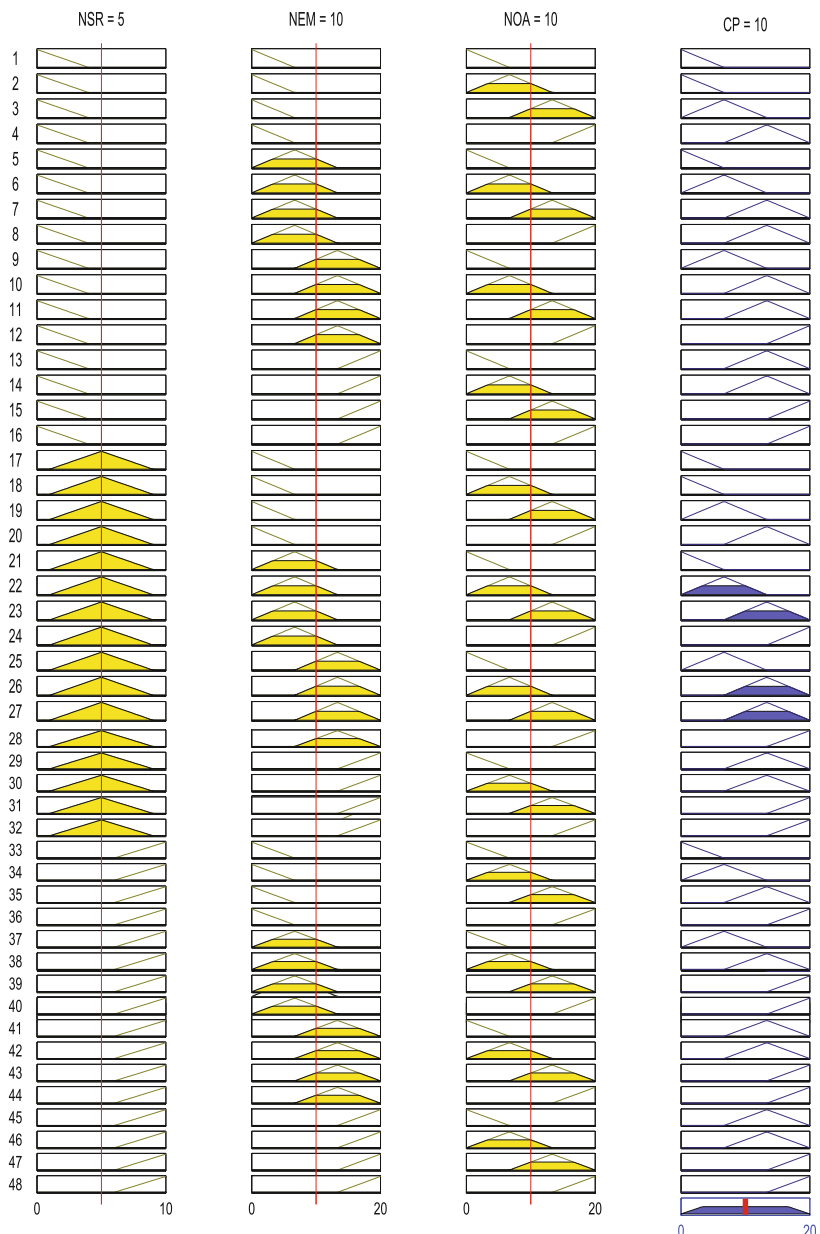

Fig. 4 Fuzzy rule view for class point calculation

fuzzy calculations, in addition to the Max-Min composition operator, the Mamdani implication operator, and the Maximum operator for aggregation and then the model is defuzzified.

The fuzzy rule viewer shown in Fig. 4 helps to calculate the entire implication process from beginning to end.

Figure 5 displays the output surface of the FIS used for class point calculation.

After calculating the final class point values, the data sets are then being normalized. While implementing the various adaptive regression techniques for software effort estimation, the normalized $\mathrm{CP} 2$ values have been taken as input argument to find out the predicted effort. Then the predicted effort values are compared with the actual effort values to estimate the accuracy of the different model. The normalized data set is divided into different subsets using double sampling procedure. In the first step the normalized dataset is divided into training set and test set. The training set is used for learning (model estimation), whereas the test set is used only for estimating the prediction risk of the final model. The second step deals with selecting the model 
Fig. 5 Surface view of rules
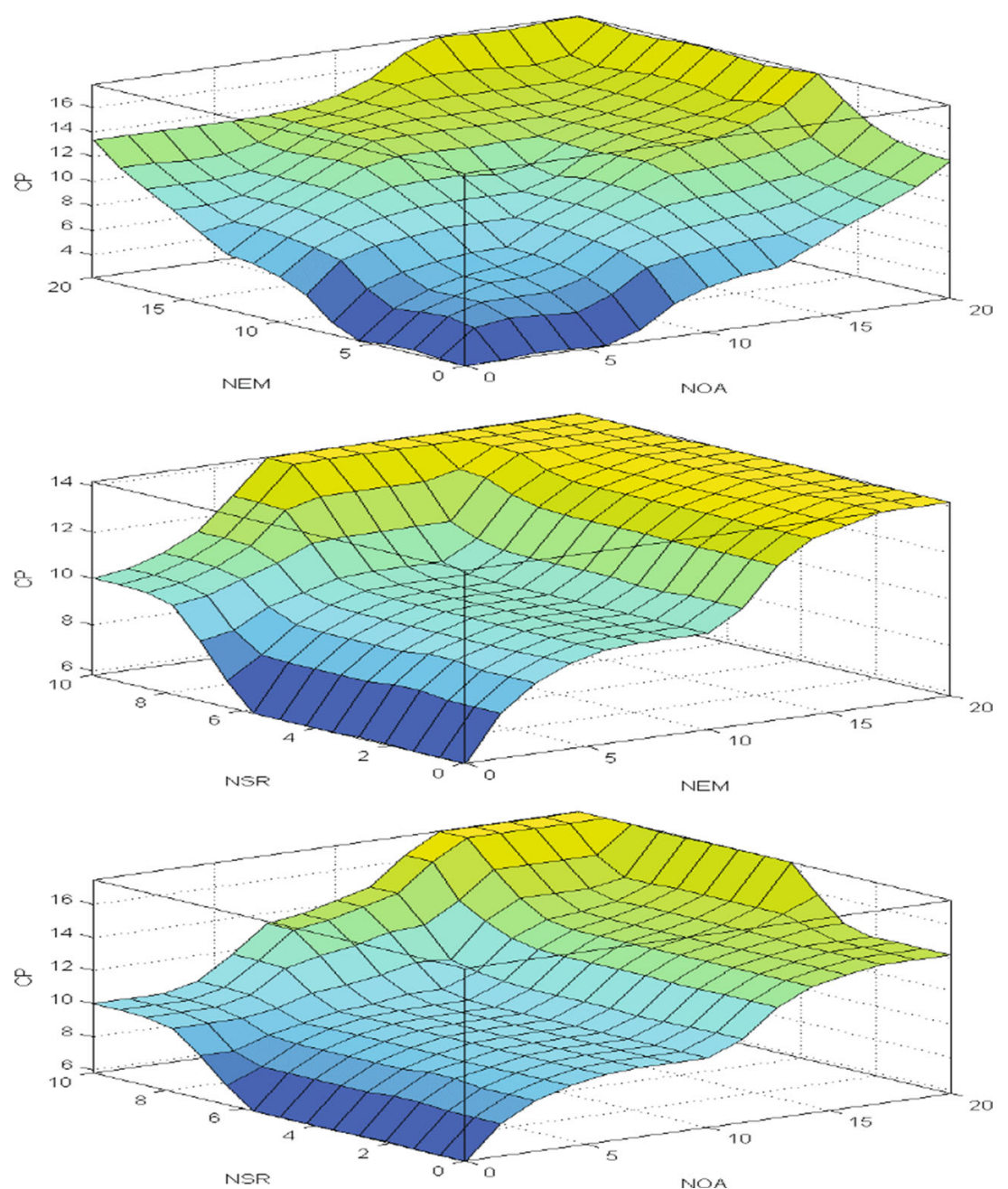

with optimal complexity. In this step, the training set is divided into learning set and validation set. The learning set is used to estimate model parameters, and the validation set is used for selecting an optimal model complexity (usually via cross-validation).

First of all, every fifth data out of 40 data, is extracted for testing purpose and rest data will be used for training purpose. Hence after the completion of fist step of double sampling process, the 40 project dataset has been divided into 32 data and eight data for preparing training and test set. Then every fifth data of training set is extracted to form validation set and rest data will be used for learning set. Hence after completion of second step of double sampling process, the complete 32 project data set has been divided into 26 data for learning and six data for validation. The fivefold data partitioning is done on the training data by the following strategy :

For partition 1: Samples 1, 6, 11,.. are used as validation samples and the remaining as learning samples
For partition 2: Samples 2, 7, 12,... are used as validation samples and the remaining as learning samples

For partition 5: Samples 5, 10, 15,... are used as validation samples and the remaining as learning samples.

After partitioning data into learning set and validation set, the model selection is performed using 5-fold crossvalidation process. The performance of the model can be evaluated by using the following criteria:

The magnitude of relative error (MRE) is a very common criterion used to evaluate software cost estimation models. The MRE for each observation i can be obtained as:

$M R E_{i}=\frac{\mid \text { ActualEffort }_{i}-\text { PredictedEffort }_{i} \mid}{\text { ActualEffort }_{i}}$

The mean magnitude of relative error (MMRE) can be achieved through the summation of MRE over $\mathrm{N}$ observations 
$M M R E=\sum_{1}^{N} M R E_{i}$

The root mean square error (RMSE) is just the square root of the mean square error.

$R M S E=\sqrt{\frac{\sum_{i=1}^{N}\left(y_{i}-\bar{y}\right)^{2}}{N}}$

The normalized root mean square (NRMS) can be calculated by dividing the RMSE value with standard deviation of the actual effort value for training data set.

$N R M S=\frac{R M S E}{\operatorname{mean}(Y)}$

where $\mathrm{Y}$ is the actual effort for testing data set.

The prediction accuracy (PA) can be calculated as:

$P A=\left(1-\left(\frac{\sum_{i=1}^{N} \mid \text { actual }- \text { predicted } \mid}{N}\right)\right) * 100$

The average of all the folds corresponding NRMSE value for training and testing set will be considered as the final NRMSE value of the model. The model producing lower NRMSE value, will be selected for testing and final comparison.

\subsection{Model design using multi-layer perceptron}

This technique uses one parameter. This parameter sets the number of hidden neurons to be used in a three layer neural network. The number of neurons used is directly proportional to the training time. The values are typically between 2 and 40, but it can be as high as 1000 . While implementing the normalized data set using Multi-Layer Perceptron technique for different number of hidden neurons, the following results have been obtained.

Table 6 provides minimum NRMSE value obtained from training set and test set using the multi-layer perceptron technique for each fold for a specific number of hidden neurons. Hence the final result will be the average

Table 6 NRMSE value obtained using multi-layer perceptron technique for different no. of neurons

\begin{tabular}{lllll}
\hline Fold & $\begin{array}{l}\text { Number } \\
\text { of hidden } \\
\text { neurons }\end{array}$ & MMRE & $\begin{array}{l}\text { Training set } \\
\text { validation error } \\
\text { (NRMSE) }\end{array}$ & $\begin{array}{l}\text { Test set } \\
\text { prediction error } \\
\text { (NRMSE) }\end{array}$ \\
\hline 1 & 15 & 0.4055 & 0.3282 & 0.2529 \\
2 & 25 & 0.2314 & 0.3654 & 0.2606 \\
3 & 20 & 0.6468 & 0.2590 & 0.5626 \\
4 & 10 & 0.1127 & 0.3117 & 0.2374 \\
5 & 30 & 1.2202 & 0.2702 & 0.3501 \\
\multicolumn{2}{l}{ Average } & 0.5233 & 0.3069 & 0.3327 \\
\hline
\end{tabular}

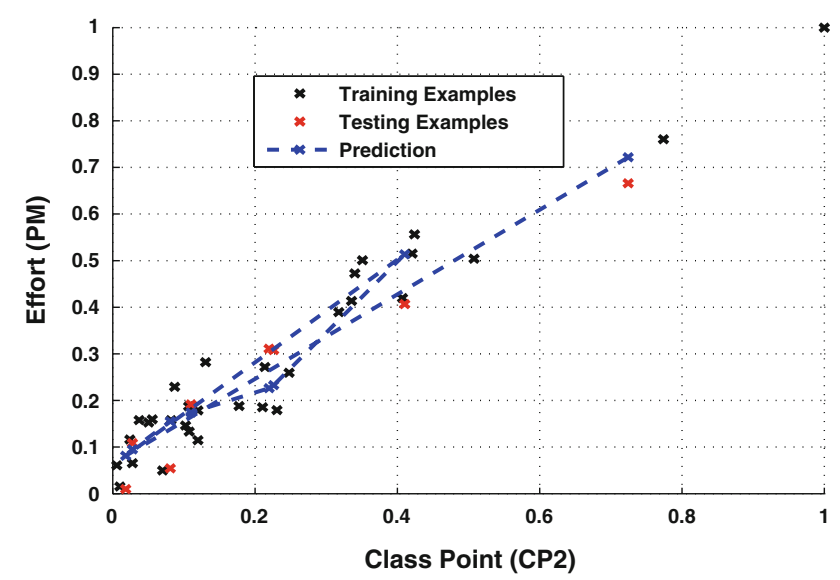

Fig. 6 Multi-Layer perceptron based effort estimation model

of the NRMSE values for training set and test set. The proposed model generated using the multi-layer perceptron technique is plotted based on the training and testing sample as shown in Fig. 6.

From the above figure, it has been observed that predicted value is highly correlated with actual data.

\subsection{Model design using multivariate adaptive regression splines}

This technique uses two principal tuning parameters. First parameter is the maximum number of basis functions to use. This parameter controls the maximum amount of complexity of the model. In most cases, the results are not very sensitive to this parameter. The range of this parameter is from 1 to 100. For most problems, this parameter can be set to a high value (50-100) which results in a good fit.

Second parameter is the amount of penalty given to complex models. This is an integer, which ranges from 0 to 9 where 0 means a small penalty is given to complex models and 9 means a large penalty is given. This parameter is adjusted based on the estimated amount of noise in the data, and has the largest effect on the results. For problems with little noise a value less than 5 are suggested. For noisy problems, values larger than 5 can be used. A good starting value for this parameter is 5 .

The model complexity is controlled (in conjunction) by both the parameters. However, setting the first parameter to a very large value allows the model complexity to be controlled by just the second parameter. In a simplified way we can say that, higher the value of second parameter, lower is the model complexity. While implementing the normalized data set using MRS technique for number of basis functions used along with the amount of penalty given to the models, the following results have been obtained.

From Table 7, it is clearly visible that the model with five no. of basis functions and zero amount of penalty 
Table 7 NRMSE value obtained using multivariate adaptive regression splines for different no. of basis functions and penalty parameters

\begin{tabular}{llllll}
\hline Fold & $\begin{array}{l}\text { Number } \\
\text { of basis } \\
\text { functions }\end{array}$ & $\begin{array}{l}\text { Amount } \\
\text { of } \\
\text { penalty }\end{array}$ & MMRE & $\begin{array}{l}\text { Training } \\
\text { set validation } \\
\text { error } \\
\text { (NRMSE) }\end{array}$ & $\begin{array}{l}\text { Test set } \\
\text { prediction } \\
\text { error } \\
\text { (NRMSE) }\end{array}$ \\
\hline 1 & 100 & 9 & 0.4042 & 0.3366 & 0.2463 \\
2 & 20 & 0 & 0.2384 & 0.2914 & 0.2640 \\
3 & 100 & 9 & 0.5852 & 0.2895 & 0.5438 \\
4 & 100 & 9 & 0.1201 & 0.3058 & 0.2478 \\
5 & 100 & 9 & 1.2239 & 0.2984 & 0.2830 \\
\multicolumn{2}{ll}{ Average } & & 0.5144 & 0.3043 & 0.3170 \\
\hline
\end{tabular}

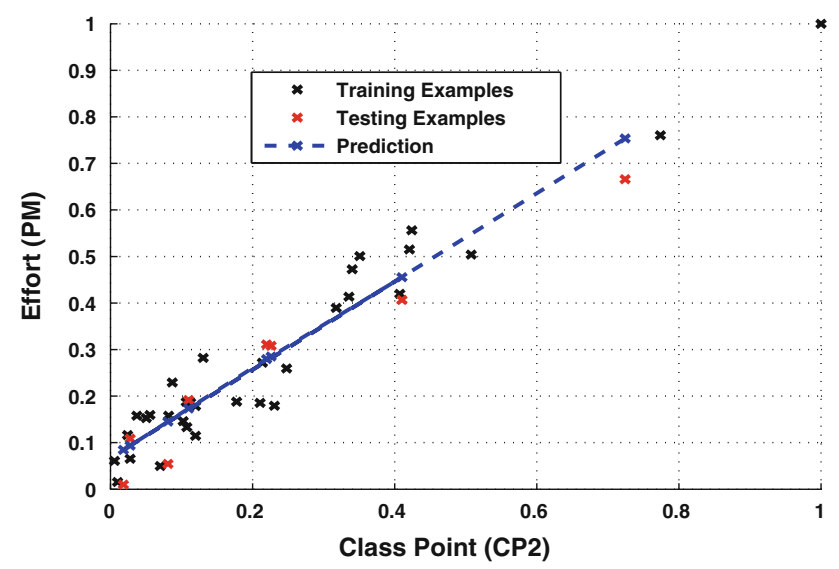

Fig. 7 Multivariate adaptive regression splines based effort estimation model

parameters, provides minimum value of NRMS and RMSE. The proposed model generated using the MRS technique is then plotted based on the training and testing sample as shown in Fig. 7.

From the above figure, it has been observed that predicted value is more highly correlated with actual data than that of obtained using MLP technique.

\subsection{Model design using project pursuit regression}

This technique requires one parameter. This parameter controls the complexity (number of terms) of the model. For most problems, a value between 1 and 10 works well. This method does not use any internal parameter selection, so this parameter directly affects the complexity of the model and must be chosen with care. While implementing the normalized data set using Project Pursuit Regression technique for different number of terms, the following results have been obtained.

Table 8, displays that the model with five no. of terms, provides minimum value of NRMS and RMSE. Figure 8 shows the proposed model generated using the project pursuit regression technique and plotted based on the training and testing sample.
Table 8 NRMSE value obtained using project pursuit regression technique for different no. of terms

\begin{tabular}{lccll}
\hline Fold & $\begin{array}{l}\text { Number } \\
\text { of terms }\end{array}$ & MMRE & $\begin{array}{l}\text { Training set } \\
\text { validation error } \\
\text { (NRMSE) }\end{array}$ & $\begin{array}{l}\text { Test set } \\
\text { prediction error } \\
\text { (NRMSE) }\end{array}$ \\
\hline 1 & 5 & 0.4064 & 0.3794 & 0.2505 \\
2 & 1 & 0.3432 & 0.3667 & 0.4108 \\
3 & 1 & 0.6038 & 0.3130 & 0.5798 \\
4 & 10 & 0.1201 & 0.3422 & 0.2440 \\
5 & 2 & 1.1695 & 0.3110 & 0.3666 \\
Average & 0.5286 & 0.3425 & 0.3703 \\
\hline
\end{tabular}

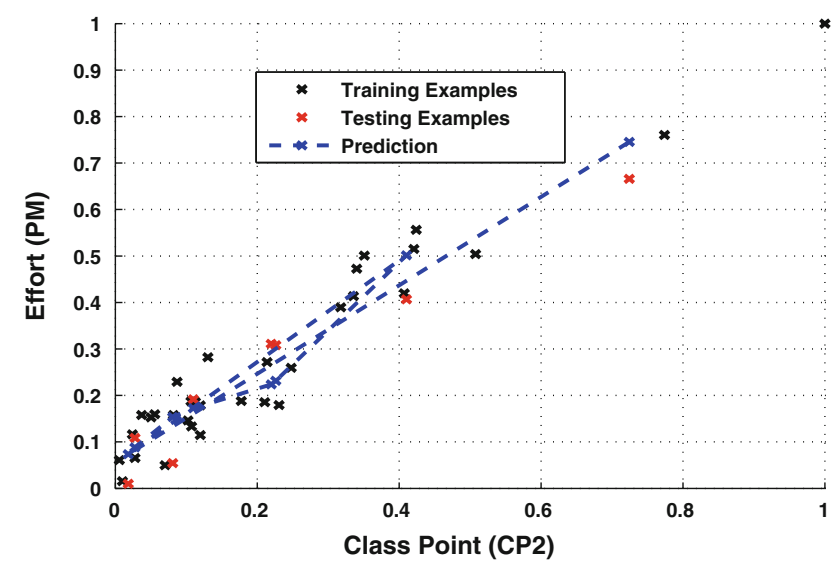

Fig. 8 Project pursuit regression based effort estimation model

From Fig. 8, it has been observed that the predicted value is less correlated with actual data.

\subsection{Model design using constrained topological mapping}

This technique uses two parameters. First parameter is the number of dimensions used in the map. This parameter is chosen based on whether constraints exit between the independent variables. It should reflect the estimated intrinsic dimensionality of the data. For most problems, this is chosen to be 1,2 , or 3 . This should always be less than or equal to the dimension of the input space.

Second parameter is a smoothing parameter. This is an integer from 0 to 9 , where 9 indicate the smoothest model. If this parameter is set to 0 then the algorithm tries to minimize the RMS error on the training set. If this parameter is set to 9 , then the algorithm tries to minimize the cross-validation error. For any value between 0 and 9 , a mixture of the two error measures is minimized. This parameter becomes critical for problems with high noise and/or small number of samples in the training set. For problems with low noise and large number of samples, the 
Table 9 NRMSE value obtained using constrained topological mapping technique for different no. of dimensions and smoothing parameters

\begin{tabular}{llllll}
\hline Fold & $\begin{array}{l}\text { Number of dimensions } \\
\text { in the map }\end{array}$ & $\begin{array}{l}\text { Smoothing } \\
\text { parameter }\end{array}$ & MMRE & $\begin{array}{l}\text { Training set validation } \\
\text { error (NRMSE) }\end{array}$ & $\begin{array}{l}\text { Test set prediction } \\
\text { error (NRMSE) }\end{array}$ \\
\hline 1 & 1 & 1 & 0.5984 & 0.3150 & 0.2526 \\
2 & 1 & 9 & 0.2150 & 0.3812 & 0.2588 \\
3 & 1 & 8 & 0.6786 & 0.3207 & 0.5466 \\
4 & 1 & 9 & 0.1189 & 0.3507 & 0.2401 \\
5 & 1 & 9 & 1.2561 & 0.3007 & 0.5319 \\
Average & & 0.5534 & 0.3337 & 0.3660 \\
\hline
\end{tabular}

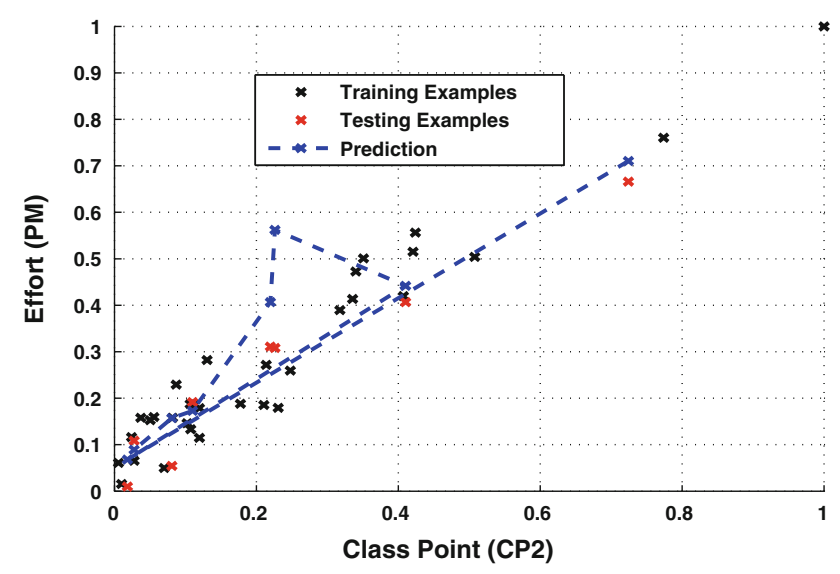

Fig. 9 Constrained topological mapping based effort estimation model

parameter has little effect. For high noise problems, a larger number is suggested.

While implementing the normalized data set using CTM technique for different number of dimensions used along with corresponding smoothing parameter value, the following results have been obtained.

From Table 9, it is clearly visible that the model with one dimension in the map and smoothing parameter value of nine, provides minimum value of NRMS and RMSE. The proposed model generated using the CTM technique is then plotted based on the basis of training and testing sample.

From Fig. 9, it has been observed that the predicted value is less correlated with actual data.

\subsection{Model design using K-nearest neighbor regression}

This technique uses one parameter called $K$ which specifies the numbers of nearest neighbors that are averaged to form an estimate. The value for $K$ must be greater than 0 but less than the number of samples in training file. The maximum value of $K$ could be 100 . While implementing the normalized data set using K-nearest neighbor regression technique for different numbers of nearest neighbors, the following results have been obtained.
Table 10 NRMSE value obtained using K-nearest neighbor regression for different no. of nearest neighbours

\begin{tabular}{lllll}
\hline Fold & $\begin{array}{l}\text { Number of } \\
\text { nearest } \\
\text { neighbours }\end{array}$ & MMRE & $\begin{array}{l}\text { Training set } \\
\text { validation error } \\
\text { (NRMSE) }\end{array}$ & $\begin{array}{l}\text { Test set } \\
\text { prediction error } \\
\text { (NRMSE) }\end{array}$ \\
\hline 1 & 2 & 0.3918 & 0.4096 & 0.2813 \\
2 & 2 & 0.3805 & 0.4375 & 0.4313 \\
3 & 4 & 0.6432 & 0.3406 & 0.6439 \\
4 & 4 & 0.1251 & 0.3738 & 0.2312 \\
5 & 2 & 1.1529 & 0.3829 & 0.3616 \\
Average & 0.5387 & 0.3889 & 0.3899 \\
\hline
\end{tabular}

Table 10 provides various estimation parameters value such as NRMS, RMSE and MMER obtained using the K-Nearest Neighbor Regression technique for different no. of nearest neighbors. From the above table, it is clear that the model with two no. of nearest neighbors provides minimum value of NRMS and RMSE. The proposed model generated using the K-Nearest Neighbor Regression technique is plotted based on the training and testing sample as shown in Fig. 10.

From the above figure, it has been observed that the predicted value is less correlated with actual data.

\subsection{Model design using radial basis function network}

This technique uses one parameter i.e., the number of basis functions. This parameter should be greater than 1. For multivariate input, this parameter should be a squared number (i.e., 4, 9, 25, 36, etc.). Moreover, this parameter should not be greater than the number of samples in the training data. While implementing the normalized data set using RBFN technique for different number of basis functions, the following results have been obtained.

Table 11 provides minimum NRMSE value obtained from Training set and Test set using the RBFN technique for each fold for a specific number of basis functions. Hence the final result will be the average of the NRMSE values obtained from training set and test set. The proposed model generated using the RBFN technique is plotted 


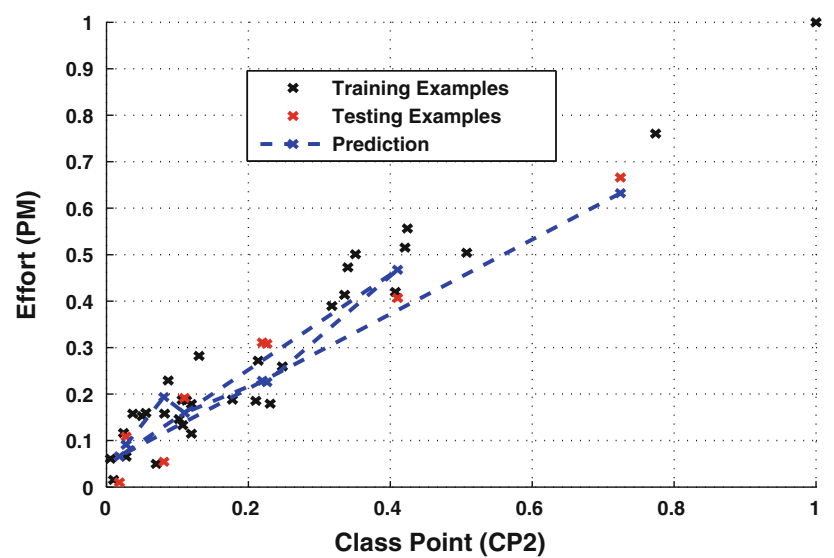

Fig. 10 K-nearest neighbor regression based effort estimation model

Table 11 NRMSE value obtained using radial basis function network technique based on no. of basis functions

\begin{tabular}{lllll}
\hline Fold & $\begin{array}{l}\text { Number } \\
\text { of basis } \\
\text { functions }\end{array}$ & MMRE & $\begin{array}{l}\text { Training set } \\
\text { validation error } \\
\text { (NRMSE) }\end{array}$ & $\begin{array}{l}\text { Test set } \\
\text { prediction error } \\
\text { (NRMSE) }\end{array}$ \\
\hline 1 & 2 & 0.3961 & 0.4525 & 0.2495 \\
2 & 2 & 0.2282 & 0.3985 & 0.3158 \\
3 & 2 & 0.6601 & 0.3546 & 0.5566 \\
4 & 2 & 0.1208 & 0.3817 & 0.2438 \\
5 & 2 & 1.2207 & 0.3411 & 0.3063 \\
\multicolumn{2}{l}{ Average } & 0.5252 & 0.3857 & 0.3344 \\
\hline
\end{tabular}

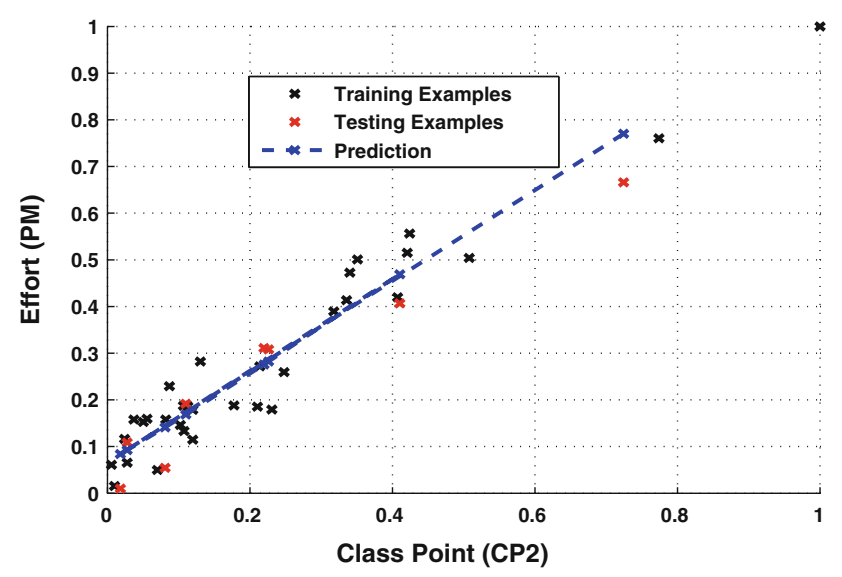

Fig. 11 Radial basis function network based effort estimation model

based on the training and testing sample as shown in Fig. 11.

From the above figure, it has been observed that the predicted value is highly correlated with actual data, but less correlation than that of obtained using MRS technique.

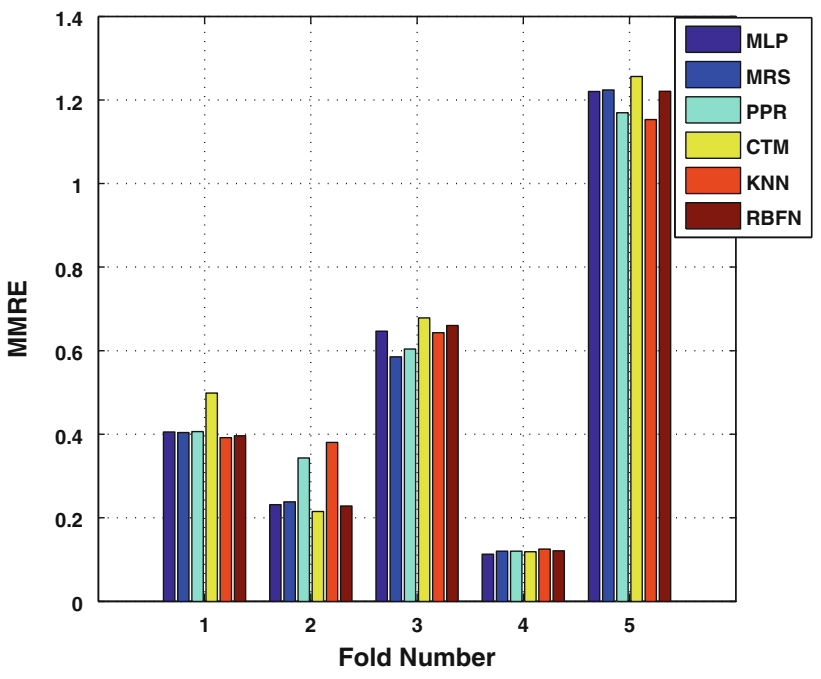

Fig. 12 Comparison of MMRE values obtained using six adaptive methods for regression

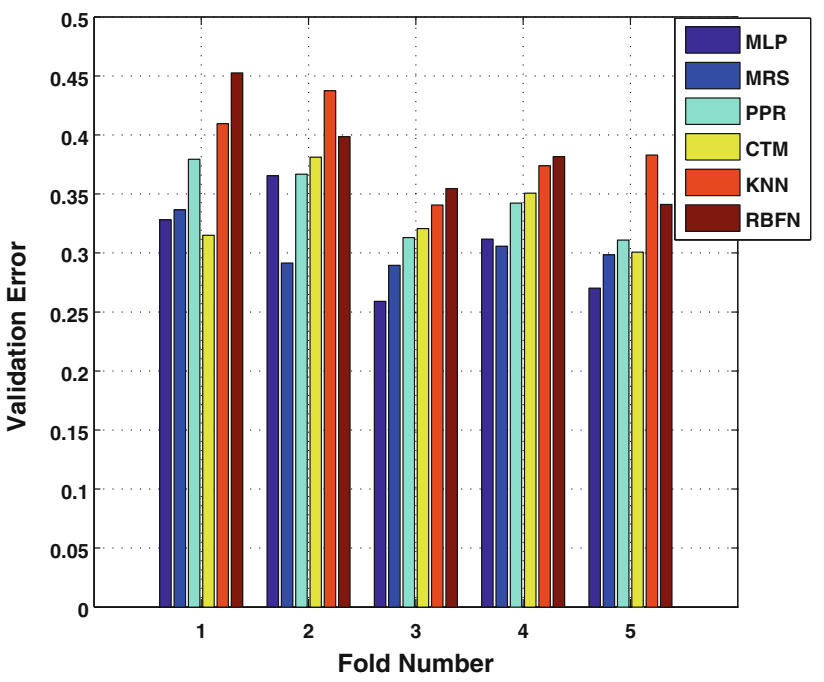

Fig. 13 Comparison of validation error obtained using six adaptive methods for regression

\subsection{Comparison}

On the basis of results obtained, the estimated effort value using the six adaptive methods for regression are compared. The results show that effort estimation using MRS gives better values of NRMSE than those obtained using other five methods.

Figure 12 shows the comparison between MMRE values obtained using six adaptive regression methods.

Figure 13 shows the comparison between validation error obtained using six adaptive regression methods.

Figure 14 shows the comparison between prediction error obtained using six adaptive regression methods. 


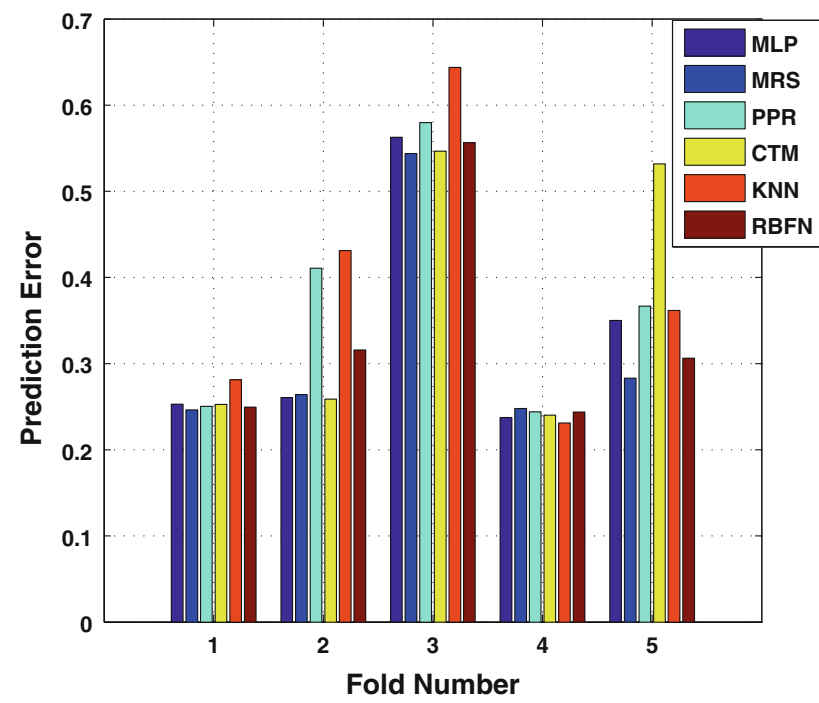

Fig. 14 Comparison of prediction error obtained using six adaptive methods for regression

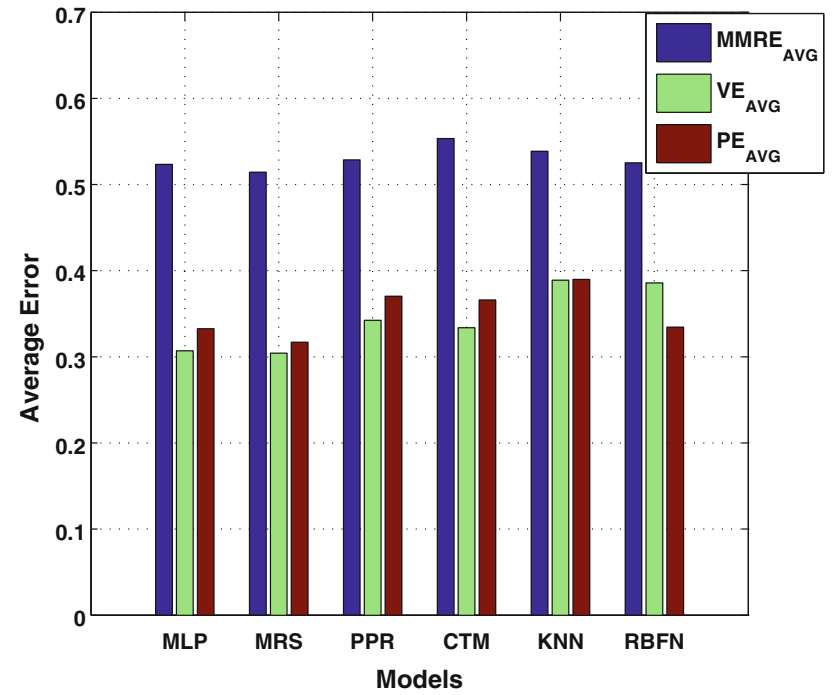

Fig. 15 Comparison of average error values obtained from training and test set using six adaptive methods for regression

Figure 15 shows the comparison of average error values obtained from training set (validation error) and test set (prediction error) for six adaptive methods for regression techniques.

When using the NRMSE, MMRE and prediction accuracy in evaluation, good results are implied by lower values of NRMSE, MMRE and higher values of prediction accuracy. Table 12 displays the final comparison of NRMSE, MMRE and prediction accuracy value for six adaptive regression methods. From the table, it is clear that the effort estimation using MRS method gives least NRMSE, MMRE and high prediction accuracy value than other five techniques. Similarly, a comparison has been made between the
Table 12 Comparison of NRMSE, MMRE and prediction accuracy values between six adaptive methods for regression

\begin{tabular}{cllll}
\hline & $\begin{array}{l}\text { Average } \\
\text { validation } \\
\text { error } \\
\text { (NRMSE) }\end{array}$ & $\begin{array}{l}\text { Average } \\
\text { prediction } \\
\text { error } \\
\text { (NRMSE) }\end{array}$ & $\begin{array}{l}\text { Average } \\
\text { MMRE }\end{array}$ & $\begin{array}{l}\text { Prediction } \\
\text { accuracy (in } \\
\text { percentage) }\end{array}$ \\
\hline $\begin{array}{c}\text { Multi-layer } \\
\text { perceptron }\end{array}$ & 0.3069 & 0.3327 & 0.5233 & 94.8185 \\
$\begin{array}{c}\text { Multivariate } \\
\text { adaptive } \\
\text { regression } \\
\text { splines }\end{array}$ & 0.3043 & 0.3170 & 0.5144 & 95.0639 \\
$\begin{array}{c}\text { Projection } \\
\text { pursuit } \\
\text { regression }\end{array}$ & 0.3425 & 0.3703 & 0.5286 & 94.0546 \\
$\begin{array}{c}\text { Constrained } \\
\text { topological } \\
\text { mapping }\end{array}$ & 0.3337 & 0.3660 & 0.5534 & 94.5322 \\
$\begin{array}{c}\text { K nearest } \\
\text { neighbor } \\
\text { regression }\end{array}$ & 0.3889 & 0.3899 & 0.5387 & 93.8137 \\
$\begin{array}{c}\text { Radial basis } \\
\text { function } \\
\text { network }\end{array}$ & 0.3857 & 0.3344 & 0.5252 & 94.7539 \\
\hline
\end{tabular}

obtained results with the work mentioned in the related work section. By analyzing results, it can be concluded that the prediction accuracy obtained by Costagliola et al. [10], Zhou and Liu [28] and Kanmani et al. [19] is 83, 83 and $87 \%$ respectively; where as the proposed techniques shows significant improvement in the accuracy values as shown in Table 12. Hence, it can be concluded that the proposed approaches outperform the techniques mentioned in the related work section.

\section{Conclusion and future work}

Several approaches have already been defined in literature for software effort estimation. But the CPA is one of the different cost estimation models that has been widely used because it is simple, fast and accurate to a certain degree. Fuzzy-logic technique is further used to find out the complexity level of the class and to calculate optimized class point. Then the calculated class point values are being normalized and used to optimize the effort estimation result. The optimal values are obtained by implementing six different types of adaptive methods of regression techniques such as ANN, MRS, PPR, CTM, KNN and RBFN using normalized class point value. Finally the generated minimum results of different techniques have been compared to estimate their performance accuracy. Result shows that MRS based effort estimation model gives less value of NRMSE, MMRE and higher value of 
prediction accuracy. Hence it can be concluded that the effort estimation using MRS model will provide more accurate results than other five techniques. The computations for above procedure have been implemented and membership functions generated using MATLAB. This approach can also be extended by using some other techniques such as Random Forest and Gradient Boosted Trees.

\section{References}

1. Albrecht AJ Measuring application development productivity. In Proceedings of the Joint SHARE/GUIDE/IBM Application Development Symposium, volume 10, pages 83-92. SHARE Inc. and GUIDE International Corp. Monterey, CA, 19;79

2. Attarzadeh I, Ow S (2010) Soft computing approach for software cost estimation. Int J Software Eng, IJSE 3(1):1-10

3. Bataineh K, Naji M, Saqer M (2011) A comparison study between various fuzzy clustering algorithms. Editorial Board, 5(4):335

4. Bors A (2001) Introduction of the radial basis function (rbf) networks. In Online symposium for electronics engineers, volume 1 , pp 1-7

5. Braga PL, Oliveira AL, Meira SR (2008) A ga-based feature selection and parameters optimization for support vector regression applied to software effort estimation. In Proceedings of the 2008 ACM symposium on Applied computing, pp 1788-1792. ACM

6. Carbone M, Santucci G (2002) Fast\&\&serious: a UML based metric for effort estimation. In: Proceedings of the 6th ECOOP workshop on quantitative approaches in object-oriented software engineering (QAOOSE'02), pp 313-322

7. Cherkassky V, Gehring D, Mulier F (1996) Comparison of adaptive methods for function estimation from samples. IEEE Trans Neural Netw 7(4):969-984

8. Cherkassky V, Lari-Najafi H (1991) Constrained topological mapping for nonparametric regression analysis. Neural Netw 4(1):27-40

9. Cherkassky V, Mulier FM (2007) Learning from data: concepts, theory, and methods. Wiley-IEEE Press, New Jersey

10. Costagliola G, Ferrucci F, Tortora G, Vitiello G (2005) Class point: an approach for the size estimation of object-oriented systems. IEEE Trans Soft Eng 31(1):52-74

11. Costagliola G, Ferrucci F, Tortora G, Vitiello G (1998) Towards a software size metrics for object-oriented systems. Proc. AQUIS 98:121-126

12. Devroye L (1978) The uniform convergence of nearest neighbor regression function estimators and their application in optimization. IEEE Trans Inf Theory 24(2):142-151
13. Drucker H, Burges CJ, Kaufman L, Smola A, Vapnik V (1997) Support vector regression machines.In Mozer M.C., Jordan M.I., and Petsche T. (eds) Advances in neural information processing systems. MIT Press, Cambridge, pp 155-161

14. Friedman JH (1991) Multivariate adaptive regression splines. Ann Stat 19:1-67

15. Friedman JH, Stuetzle W (1981) Projection pursuit regression. J Am Stat Assoc 76(376):817-823

16. Heemstra F, Kusters R (1991) Function point analysis: evaluation of a software cost estimation model. Eur J Inf Syst 1(4):229-237

17. Idri A, Abran A, Mbarki S (2006) An experiment on the design of radial basis function neural networks for software cost estimation. In Information and Communication Technologies, 2006. ICTTA'06. 2nd, vol 1, IEEE, pp 1612-1617.

18. Kanmani S, Kathiravan J, Kumar SS, Shanmugam M (2008) Class point based effort estimation of $\mathrm{OO}$ systems using fuzzy subtractive clustering and ANNs. In Proceedings of the 1st India software engineering conference, ISEC '08, ACM, pp 141-142, New York, NY, USA, 2008

19. Kanmani S, Kathiravan, J, Senthil Kumar S, Shanmugam M (2007) Neural network based effort estimation using class points for OO systems. In Computing: Theory and Applications, 2007. ICCTA'07. International Conference on IEEE, pp 261-266

20. Kim S, Lively W, Simmons D (2006) An effort estimation by UML points in early stage of software development. In: Proceedings of the international conference on software engineering research and practice, pp 415-421

21. Klair AS, Kaur RP (2012) Software effort estimation using SVM and KNN. In international conference on computer graphics, simulation and modeling (ICGSM2012), pp 146-147

22. Matson JE, Barrett BE, Mellichamp JM (1994) Software development cost estimation using function points. IEEE Trans Soft Eng 20(4):275-287

23. Oliveira AL (2006) Estimation of software project effort with support vector regression. Neurocomputing 69(13):1749-1753

24. Panchapakesan C, Palaniswami M, Ralph D, Manzie C (2002) Effects of moving the center's in an rbf network. IEEE Trans Neural Netw 13(6):1299-1307

25. S Moertini V (2008) Introduction to five data clustering algorithms. INTEGRAL Majalah Ilmiah Matematika dan Ilmu Pengetahuan Alam 7(2)

26. Sarimveis H, Alexandridis A, Bafas G (2003) A fast training algorithm for rbf networks based on subtractive clustering. Neurocomputing 51:501-505

27. Smola AJ, Schölkopf B (2004) A tutorial on support vector regression. Stat Comput 14(3):199-222

28. Zhou W, Liu Q (2010) Extended class point approach of size estimation for $\mathrm{OO}$ product. In 2nd international conference on computer engineering and technology (ICCET), 2010, vol 4, IEEE pp $117-122$ 